\title{
Mise en évidence de mâles hétérozygotes chez l'hyménoptère Diadromus pulchellus (Ichneumonide)
}

\author{
M.P. HEDDERWICK, M. EL AGOZE, P. GARAUD et G. PERIQUET \\ Institut de Biocénotique expérimentale des Agrosystèmes, \\ U.A. C.N.R.S. 340, Parc Grandmont, F 37200 Tours
}

\begin{abstract}
Résumé
Chez l'hyménoptère Diadromus pulchellus à parthénogenèse arrhénotoque, les mâles sont normalement haploïdes. L'étude du polymorphisme enzymatique aux loci Pgm-2 et Ao-4, d'individus provenant d'une population du sud de la France et étudiée au laboratoire durant les 5 premières générations, a mis en évidence la présence de mâles hétérozygotes à ces 2 loci. L'analyse caryologique a montré que ces individus étaient de fait totalement diploïdes. Au laboratoire des mâles hétérozygotes parfaitement viables ont été obtenus par un ensemble de croisements consanguins et se sont également révélés diploïdes. L'existence de ce type de mâles pose le problème de leur origine et de leur impact sur l'évolution des populations de cet hyménoptère.
\end{abstract}

Mots clés : Polymorphisme enzymatique, caryotype, consanguinité.

\section{Summary \\ Heterozygous males in D. pulchellus}

Individual males of the hymenoptera Diadromus pulchellus are normally haploid. Studies of enzyme polymorphism at the Pgm-2 and Ao-4 loci of individuals sampled from a French southern population and analysed during the first 5 generations in the laboratory showed the existence of viable heterozygous males at those loci. Caryological analysis of these males revealed their totally diploid structure. In laboratory conditions, heterozygous males have been obtained by inbreeding and shown to be diploid. Their existence raises the question of their origin and their impact on the population dynamics of this hymenoptera.

Key words : Enzymatic polymorphism, caryotype, inbreeding.

\section{Introduction}

Le mode de reproduction fondamental des hyménoptères est basé sur la parthénogenèse arrhénotoque donnant normalement des mâles haploïdes, tandis que les femelles (diploïdes) résultent de la fécondation d'un ovocyte (revue in Crozier, 1975). Chez Diadromus pulchellus, un endoparasite solitaire des chrysalides de la teigne du poireau (Acrolepiopsis assectella) (LABEYRIE, 1959, 1964 ; RoJAS-Rousse, 1975, 1977, 1981), 
l'étude de populations naturelles et expérimentales a révélé la présence de mâles diploïdes. Ces résultats sont comparés à ceux obtenus chez d'autres espèces d'hyménoptères.

\section{Matériel et méthodes}

Les parasites fondateurs (19 femelles et 13 mâles), capturés en septembre 1983 dans un champ cultivé en Allium porrum au plan de Grasse, vallée de la Siagne (Alpes-Maritimes, France), ont été mis en cage à populations (Souche $\mathrm{S} \mathrm{83)}$ et maintenus au laboratoire dans les conditions suivantes : thermopériode $25^{\circ} \mathrm{C}-15^{\circ} \mathrm{C} \pm 1{ }^{\circ} \mathrm{C}$, humidité relative 70 p. $100 \pm 10$ p. 100 , photopériode $16 \mathrm{~h}-8 \mathrm{~h}$. Les individus analysés ont été pris parmi les fondateurs de cette souche et parmi les descendants des 5 premières générations.

L'analyse enzymatique a été réalisée en utilisant les techniques standards pour gel d'amidon avec système tampon discontinu Tris-citrate (PoulıK, 1957).

Les systèmes enzymatiques étudiés phosphoglucomutase (PGM) et Aldéhyde oxydase (AO) ont été révélés respectivement selon les méthodes de Selander (1971) et Ayala et al. (1974).

Deux loci ont été analysés pour la phosphoglucomutase (Pgm-1 et Pgm-2), mais le locus Pgm-1 faiblement polymorphe n'a pu être utilisée. Parmi les loci codant une aldéhyde oxydase seul le quatrième (Ao-4) était suffisamment polymorphe pour cette étude.

L'analyse génétique a été conduite en utilisant la souche mutante $\mathrm{J} 82$ de corps jaune, homozygote pour l'allèle $\mathrm{j}$ récessif et la souche sauvage de référence $\mathbf{M} 72-83$ maintenue au laboratoire depuis 1972 et homozygote pour l'allèle $\mathrm{j}^{+}$déterminant un phénotype (corps noir). Des croisements entre ces 2 souches et des rétrocroisements sur la souche $\mathbf{J}$ ont été réalisés afin d'obtenir des individus consanguins étudiés aux niveaux génétique et cytogénétique.

L'analyse caryotypique a porté sur les cellules en mitose des testicules et ovarioles d'individus adultes. Ces organes prélevés par dissection des insectes dans du sérum physiologique, ont été traités par la colchicine à 0,02 p. 100 dans $\mathrm{NaCl} 9 \mathrm{p} .1000$ pendant 1 heure. Les préparations ont ensuite subi un choc hypotonique dans une solution de citrate de sodium 1 p. 100 pendant $10 \mathrm{~min}$., et une fixation dans le Carnoy I. Après écrasement des organes entre lame et lamelle, les chromosomes ont été colorés par les techniques classiques au Giemsa (Dutrillaux \& Couturier, 1981).

\section{Résultats et discussion}

Dans la souche Siagne 83, sur les 81 femelles analysées la fréquence de l'allèle rapide de la PGM $\left(\mathrm{Pgm}_{\mathrm{F}}\right)$ est de 0,593 $\pm 0,076$ et celle de l'allèle rapide de l'AO-4 (Ao-4F) est de $0,411 \pm 0,076$. Ces fréquences ne sont pas significativement différentes de celles estimées chez les mâles : $\mathrm{Pgm}^{\mathrm{F}}=0,630 \pm 0,060$, Ao-4F $=0,390 \pm 0,061 \quad(\mathrm{~N}=247)$. Parmi ces mâles $0,049 \pm 0,027$ se sont révélés hétérozygotes pour la PGM et $0,038 \pm 0,024$ pour l'AO. 


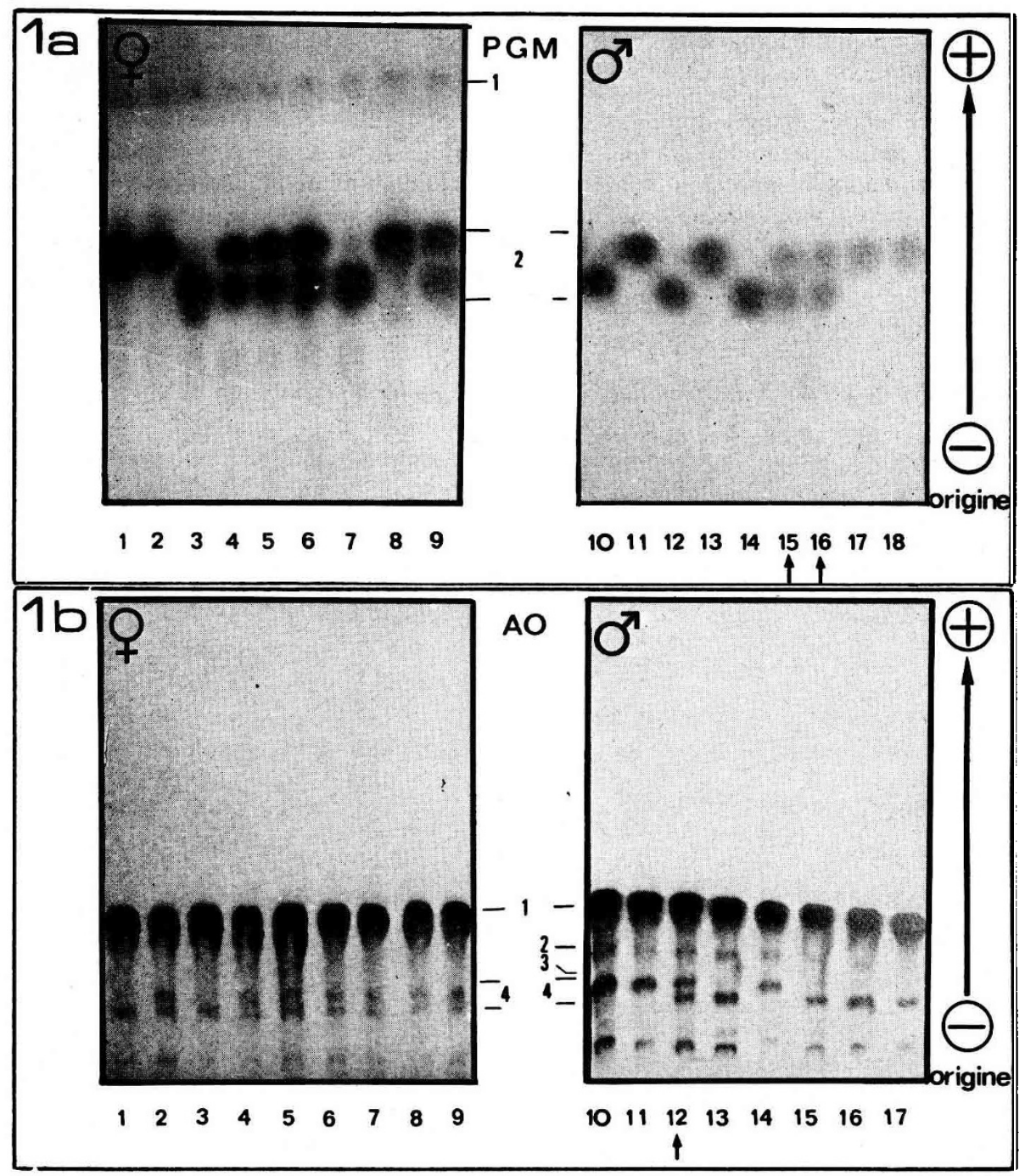

FIG. 1

Analyse électrophorétique chez D. pulchellus. Electrophoretic analysis of $\mathrm{D}$. pulchellus.

FIG. 1 a : Electrophorégramme de la phosphoglucomutase (PGM-2). Electrophoregram of phosphoglucomutase (PGM-2).

FIG. $1 \mathrm{~b}$ : Electrophorégramme de l'aldéhyde oxydase (AO-4). Les flèches indiquent la présence d'individus hétérozygotes mâles. Electrophoregram of aldehyde oxydase (AO-4). Arrows show heterozygote males. 
La figure 1 présente un électrophorégramme mettant en évidence l'existence de mâles hétérozygotes pour la phosphoglucomutase et pour l'aldéhyde oxydase. La mise en évidence de cette hétérozygotie soulève le problème du niveau de ploïdie des mâles de D. pulchellus, connus jusqu'à présent comme uniquement haploïdes (Rousse, 1980).

Des analyses cytogénétiques et enzymatiques ont été réalisées simultanément pour 15 mâles choisis parmi les descendants de la $3^{e}$ génération $(10$ homozygotes pour les 2 loci enzymatiques étudiés, et 5 hétérozygotes pour au moins un de ces loci) (Tabl. 1). Le caryotype d'une quinzaine de femelle de la souche a de plus été étudié.

\section{TABLEAU 1}

Analyse cytogénétique et enzymatique combinée des mâles de Diadromus pulchellus $(n=11)$ de la souche Siagne 83.

Combined cytogenetic and enzyme analysis of Diadromus pulchellus males $(n=11)$. (Siagne 83 strain).

\begin{tabular}{c|c|c|c}
\hline$N^{\circ}$ mâle & PGM-2 & AO-4 & Ploïdie \\
\hline 1 & FF & FF & n \\
2 & FF & FF & $2 \mathrm{n}$ \\
3 & FF & SS & n \\
4 & SS & FF & n \\
5 & SS & FF & n \\
6 & FF & FF & n \\
7 & FF & FF & $2 \mathrm{n}$ \\
8 & SS & FF & n \\
9 & SS & SS \\
10 & FF & SS & $2 \mathrm{n}$ \\
11 & FS & FS & $2 \mathrm{n}$ \\
12 & FF & FS & $2 \mathrm{n}$ \\
13 & SS & FS & $2 \mathrm{n}$ \\
14 & SS & SS & $2 \mathrm{n}$ \\
\hline
\end{tabular}

Les résultats de l'étude cytogénétique ont montré que toutes les femelles possédaient 22 chromosomes $(2 n)$ (fig. 2). Le tableau 1 résume les résultats de l'analyse combinée (cytogénétique - électrophorèse enzymatique) des mâles. On observe que la majorité des mâles monomorphes pour les loci enzymatiques étudiés possédaient un nombre haploïde de chromosomes $(n=11)$ (fig. 3), cependant 2 de ces mâles (mâles $\mathbf{n}^{\circ} 2$ et 8 ) se sont révélés diploïdes $(2 \mathrm{n}=22)$. Les 5 mâles "hétérozygotes 》 (mâles $11-15$ ) possédaient tous 22 chromosomes ( $2 \mathrm{n}$ ) (fig. 4). Aucune différence n'a été observée entre la structure chromosomique de ces mâles et celle des femelles. 

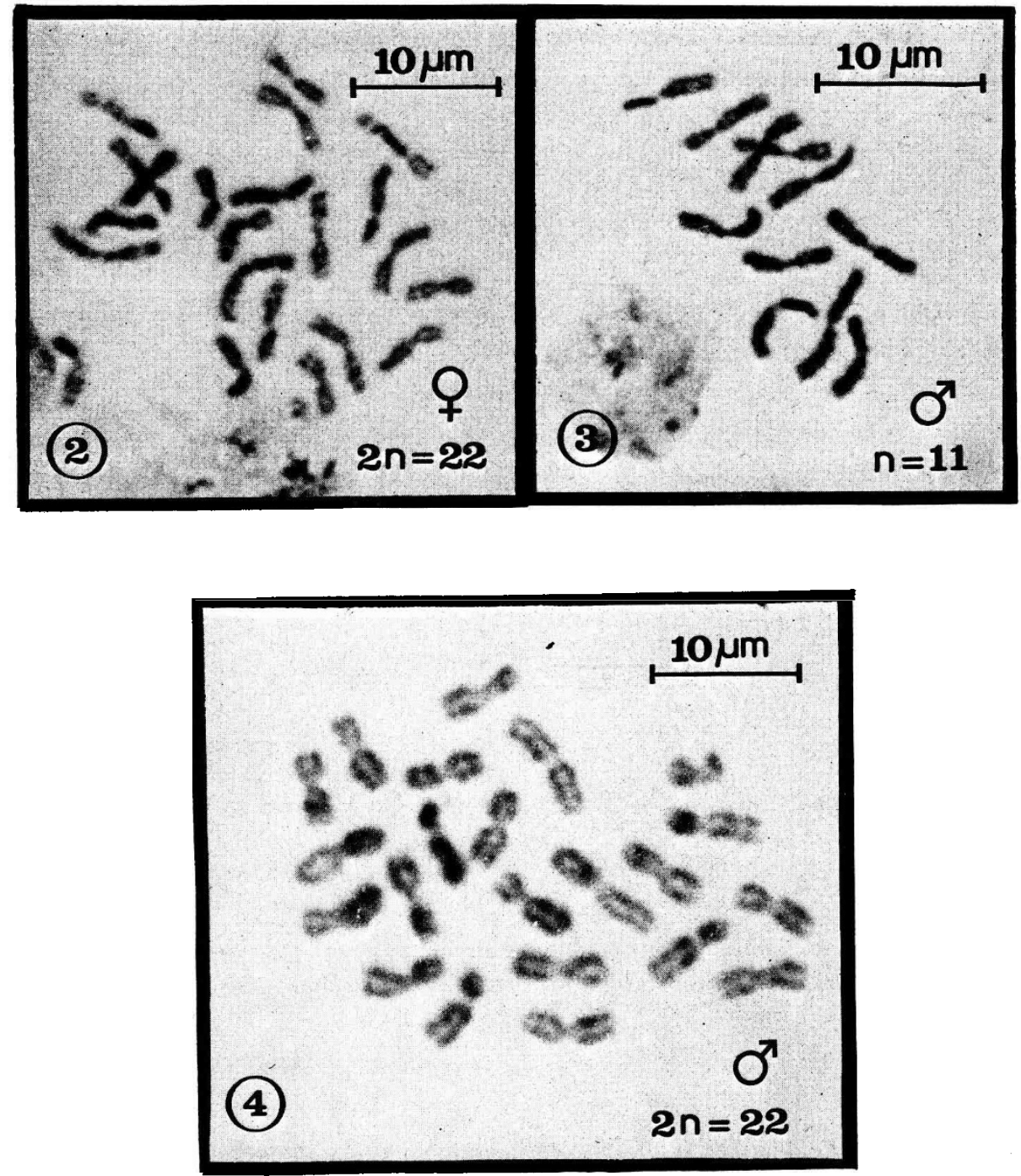

FIG. 2-4

Analyse cytogénétique de $\mathrm{D}$. pulchellus.

Cytogenetic analysis of D. pulchellus.

Fig. 2 : Métaphase mitotique chez une femelle $(G=1700 \times) ; 2 n=22$. Mitotic metaphase of female $(G=1700 \times) ; 2 n=22$.

FIG. 3 : Métaphase mitotique chez un mâle haploïde $(G=2000 \times) ; n=11$. Mitotic metaphase of haploïd male $(G=2000 \times) ; n=11$.

FIG. 4 : Métaphase mitotique $(G=1800 \times)$ chez un mâle diplö̈de; $2 n=22$. Mitotic metaphase $(G=1800 \times)$ of diploid male; $2 n=22$. 
Compte tenu de l'approche utilisée nous ne pouvons cependant mettre en évidence d'autres phénomènes cytogénétiques pouvant causer l'hétérozygotie des mâles, hormis leur diploïdisation totale qui en rend compte à elle seule.

SOUCHE M72-83

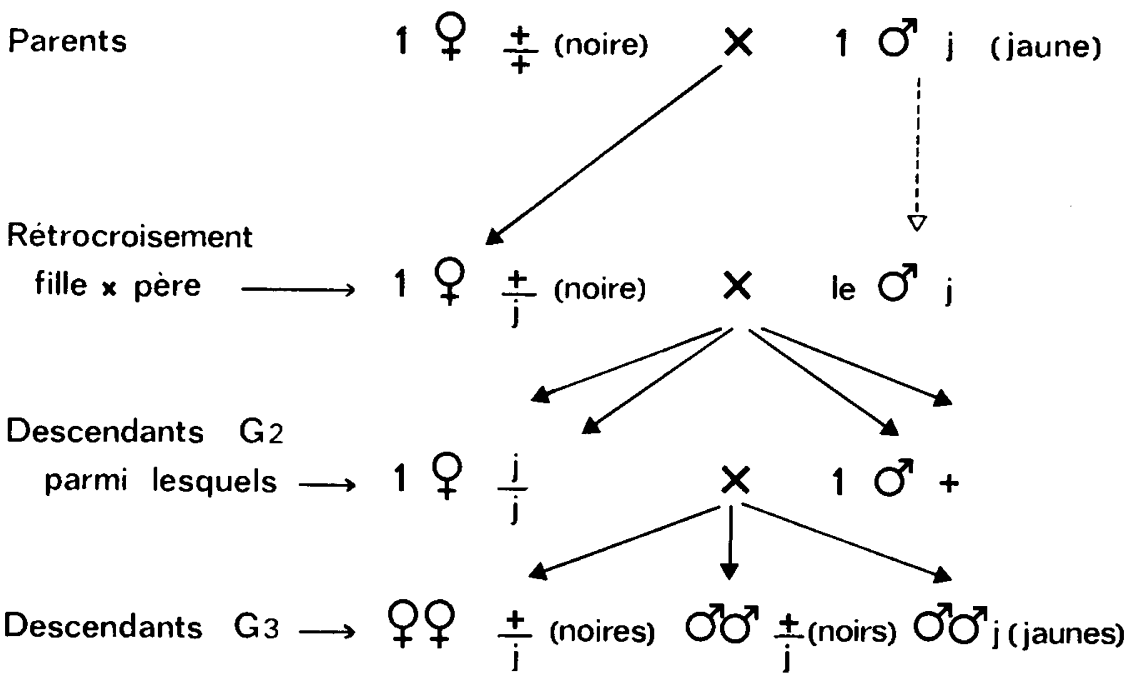

FIg. 5

Protocole de croisement permettant d'obtenir des mâles hétérozygotes au locus j. Diagram of crosses leading to heterozygous males at the $j$ locus.

TABleau 2

Résultats de la $3^{e}$ génération de croisements consanguins permettant l'obtention de mâles hétérozygotes.

Results of the 3rd inbred generation leading to heterozygous males.

\begin{tabular}{c|c|c|c|c}
\hline \multicolumn{5}{c|}{ Nombre de descendants } \\
\hline \multirow{2}{*}{ Repliques } & $\begin{array}{c}\text { Nombre } \\
\text { de croisements }\end{array}$ & $\begin{array}{c}\text { Femelles } \\
\text { (noires) }\end{array}$ & $\begin{array}{c}\text { Mâles (noirs) } \\
\text { (hétérozygotes) }\end{array}$ & $\begin{array}{c}\text { Mâles } \\
\text { (jaunes) }\end{array}$ \\
\cline { 2 - 5 } 1 & 7 & 23 & 25 & 11 \\
2 & 18 & 22 & 20 & 51 \\
3 & 26 & 73 & 69 & 95 \\
4 & 30 & 43 & 41 & 78 \\
$\Sigma$ & 81 & 161 & 155 & 235 \\
\hline
\end{tabular}


L'obtention en laboratoire de mâles hétérozygotes a ensuite été recherchée afin d'examiner leur nombre de chromosomes. La figure 5 présente le protocole des croisements permettant d'obtenir et de repérer des mâles hétérozygotes pour le locus jaune. Un couple : $o \mathrm{j}^{+} / \mathrm{j}^{+}$(corps noir) $\times \hat{o} \mathrm{j}$ (corps jaune) donne naissance à des filles hétérozygotes $j+/ j$ (corps noir). L'une d'entre elles est croisée en retour avec son père et fournit des descendants femelles et mâles au corps noir ou au corps jaune. D'une femelle jaune $(j / j)$ ainsi obtenue, croisée par l'un de ses frères noirs, naissent des filles noires $(j+/ j)$, des fils jaunes $(j)$ et des fils noirs $(j+/ j)$ parfaitement viables (tabl. 2 ).

Quatre expériences indépendantes ont fourni un total de 161 femelles noires pour ' 155 mâles noirs et 235 mâles jaunes. Le caryotype de 40 de ces mâles $(21$ noirs et 19 jaunes) a été établi. Tous les mâles jaunes étaient haploïdes et tous les mâles noirs diploïdes. Parmi ces derniers aucun n'a présenté de cellules ayant un nombre de chromosomes supérieurs à 22. Dans quelques rares préparations certaines cellules ont révélé des nombres légèrement inférieurs (de 19 à 21) probablement dus à des pertes occasionnelles en cours de traitement.

\title{
IV. Conclusion
}

En conclusion, tant dans les populations naturelles qu'en laboratoire, la présence de mâles hétérozygotes a pu être mise en évidence chez Diadromus pulchellus, et cette hétérozygotie a été systématiquement correlée avec la diploïdisation totale de leur génome.

On notera que des mâles diploïdes ont été mis en évidence dans seulement neuf genres d'hyménoptères appartenant à des familles différentes (CROZIER, 1977) mais que Diadromus pulchellus est la première espèce de la famille des ichneumonidés pour laquelle ce phénomène est décrit. Il augmente ainsi notre information sur ce groupe important et pose la question des conséquences de la présence de ce type de mâles sur l'évolution des populations naturelles de cette espèce. D'autre part, compte tenu de la possibilité d'obtention de ces mâles en laboratoire, ces résultats permettront d'aborder le problème du déterminisme du sexe chez cet hyménoptère à parthénogenèse arrhénotoque.

\author{
Reçu le 12 juin 1984. \\ Accepté le 31 janvier 1985.
}

\section{Références bibliographiques}

Ayala F.J., Tracey M.L., Barr L.G., Ehrenfeld J.G., 1974. Genetic and reproductive differenciation of the subspecies Drosophila equinoxialis caribbensis. Evolution, 28, 24-41.

Baker B.S., Belote J.M., 1983. Sex determination and dosage compensation in Drosophila melanogaster. Ann. Rev. Genet., 17, 345-393.

Crozier R.H., 1975. Animal cytogenetics. 3 Vol. : Insecta 7 : Hymenoptera. 95 pp., Gebrüder Borntraeger Berlin, Stuttgart. 
Crozier R.H., 1977. Evolutionary Genetics of Hymenoptera. Ann. Rev. Entomol., 22, 263-288.

CUNHA A.B., KerR W.E., 1957. A genetical theory to explain sex-determination by arrhenotokous parthenogenesis. Forma et Functio, 1, 33-36.

Dutrillaux B., Couturier J., 1981. La pratique de l'analyse chromosomique. 87 pp. Techniques de laboratoire $n^{\circ} 12$, Masson, Paris.

KERR W.E., 1974. Advances in cytology and genetics of bees. Ann. Rev. Entomol., 19, 253-268

LABeYRIE V., 1959. Sur la fécondité des femelles vierges de Diadromus pulchellus Wsm. Bull. Soc. Entomol. France, 64, 58-60.

LABEYRIE V., 1964. Action sélective de la fréquence de l'hôte utilisable (Acrolepia assectella ZELL.) sur Diadromus pulchellus Wsm. (Hymenoptera, Ichneumonidae) : la variabilité de la fécondité en fonction de l'intensité de la stimulation. C.R. Acad. Sc. Paris, 259, 3644-3647.

Poulix M.D., 1957. Starch electrophoresis in a discontinuous system of buffers. Nature, $180,1477$.

Rojas-Rousse D., 1975. Etude chez Diadromus pulchellus W. (Hymenoptera, Ichneumonidae) parasite d'Acrolepia assectella (Lepidoptera, Plutellidae), de la durée de la copulation et et de l'influence du temps de l'insémination sur la descendance. Bull. Soc. Zool. France, 100, 235-241.

Rojas-Rousse D., 1977. Influence de l'élimination des parasites en surnombre sur la survie de la descendance des femelles vierges de Diadromus pulchellus (Hyménoptère, Ichneumonide). Ext. Exp. Appl., 21, 38-50.

Rousse D., 1980. Examen de quelques problèmes posés à propos des fluctuations du taux sexuel chez un hyménoptère entomophage Diadromus pulchellus (Ichneumonidae). Thèse Docteur ès-Sciences. Université de Tours.

Rojas-Rousse D., 1981. Conséquences d'une augmentation des températures dans un cycle thermopériodique sur la reproduction d'entomophages parasitoïdes. Bull. Soc. Zool. France, 106, 407-417.

SELANDER R.K., 1971. Biochemical polymorphism and systematics in the genus Peromyscus. I : variation in the old-field mouse (Peromyscus polionotus). Studies Genet VI, Univ. Texas Publ., 7103, 49-90. 